\title{
Brain areas involved in spatial working memory
}

\author{
Marieke van Asselen ${ }^{\text {a,c, } * \text {, Roy P.C. Kessels }}{ }^{\text {a,b }}$, Sebastiaan F.W. Neggers ${ }^{\text {a }}$, \\ L. Jaap Kappelle ${ }^{b}$, Catharina J.M. Frijns ${ }^{b}$, Albert Postma ${ }^{a}$ \\ a Psychological Laboratory, Helmholtz Instituut, Utrecht University, \\ Heidelberglaan 2, NL-3584 CS Utrecht, The Netherlands \\ ${ }^{\mathrm{b}}$ Department of Neurology, University Medical Center Utrecht, The Netherlands \\ ${ }^{\mathrm{c}}$ IBILI, Faculty of Medicine, University of Coimbra, Portugal \\ Accepted 3 October 2005 \\ Available online 21 November 2005
}

\begin{abstract}
Spatial working memory entails the ability to keep spatial information active in working memory over a short period of time. To study the areas of the brain that are involved in spatial working memory, a group of stroke patients was tested with a spatial search task. Patients and healthy controls were asked to search through a number of boxes shown at different locations on a touch-sensitive computer screen in order to find a target object. In subsequent trials, new target objects were hidden in boxes that were previously empty. Within-search errors were made if a participant returned to an already searched box; between-search errors occurred if a participant returned to a box that was already known to contain a target item. The use of a strategy to remember the locations of the target objects was calculated as well. Damage to the right posterior parietal and right dorsolateral prefrontal cortex impaired the ability to keep spatial information 'on-line', as was indicated by performance on the Corsi Block-Tapping task and the within-search errors. Moreover, patients with damage to the right posterior parietal cortex, the right dorsolateral prefrontal cortex and the hippocampal formation bilaterally made more between-search errors, indicating the importance of these areas in maintaining spatial information in working memory over an extended time period.
\end{abstract}

(C) 2005 Elsevier Ltd. All rights reserved.

Keywords: Spatial working memory; Stroke patients; Prefrontal cortex; Posterior parietal cortex; Hippocampal formation

\section{Introduction}

Working memory is an important cognitive process, in which information is held active during a short time period. It is thought to be a multi-component system, including two subsidiary slave systems, the 'phonological loop' and the 'visuospatial sketchpad' that hold limited amounts of information active, respectively of verbal and visuospatial nature (Baddeley, 2002). These slave systems are under attentional control of the 'central executive system', which is responsible for efficient planning and organizing. Over the last years, much attention has been paid to the areas in the brain that are involved in keeping information active in working memory, specifically the prefrontal cortex (Fletcher \& Henson, 2001). Different parts of the prefrontal cortex are thought to be involved in specific working-memory

\footnotetext{
* Corresponding author. Tel.: +351 239480220; fax: +351 239480280

E-mail address: masselen@ibili.uc.pt (M. van Asselen).
}

processes. For example, keeping object information active in working memory would rely on ventral areas of the lateral prefrontal cortex, whereas processing spatial information is thought to depend on dorsal areas of the lateral prefrontal cortex, as has been shown in animal studies (e.g. Wilson, Scalaidhe, \& Goldman-Rakic, 1993), functional Magnetic Resonance Imaging (fMRI) studies in children (Nelson et al., 2000) and fMRI studies in adults (Smith \& Jonides, 1997). Furthermore, neuroimaging studies have shown that in addition to the dorsolateral prefrontal cortex, the posterior parietal cortex may play a role in spatial working memory as well (Jonides et al., 1993; Nelson et al., 2000; Walter et al., 2003). In monkeys performing a spatial working memory task, concurrent metabolic activation of the parietal and prefrontal cortex has been observed (Friedman \& Goldman-Rakic, 1994), suggesting that these are important areas of the previously hypothesized network mediating spatial working memory (Goldman-Rakic, 1988). In humans, several studies have also shown concurrent activation of the prefrontal and parietal cortices in working memory (e.g. Constantinidis \& 
Wang, 2004; Glabus et al., 2003; Jonides et al., 1993; Owen et al., 1998).

Patient studies have provided further information about brain areas involved in spatial working memory. These studies have not only corroborated the involvement of the left and right prefrontal cortex in spatial working memory (Miotto, Bullock, Polkey, \& Morris, 1996; Owen, Downes, Saahakian, Polkey, \& Robbins, 1990), but have also implicated a role for the medial temporal lobe, in particular the hippocampus. Presumably, this brain area is involved in the transition of information from working memory into long-term memory. Patients with a lesion in the right medial temporal lobe have been shown to be impaired on a spatial search task (Abrahams, Pickering, Polkey, \& Morris, 1997; Feigenbaum, Polkey, \& Morris, 1996), which appeared predominantly the result of damage to the right hippocampal region (Abrahams et al., 1999). However, Kessels, Hendriks, Schouten, Van Asselen, and Postma (2004) showed that patients with left or right amygdalohippocampectomy were not impaired on any aspect of spatial working memory as measured with a search task, indicating a limited role for the hippocampal formation in spatial working memory (see also Bohbot et al., 2002).

Thus, although neuroimaging and patient studies have stressed the importance of brain areas such as the dorsolateral prefrontal cortex (DLPFC), the posterior parietal cortex (PPC) and possibly the hippocampal formation (HF), the exact role of these areas in humans remains unclear. Therefore, the goal of the present study was to further examine the importance of these areas in spatial working memory by examining patients with focal lesions caused by cerebral stroke. A spatial search task (Van Asselen, Kessels, Wester, \& Postma, 2005) was used, requiring subjects to search for target objects that are hidden in a number of boxes shown on a computer screen. Importantly, this task enables us to distinguish between processes that are related to keeping spatial information 'online' in memory over a very short time period (i.e. tapping on the visuospatial sketchpad) and the transfer of information from working memory into long-term memory. Moreover, the use of a strategy that can be applied to relieve the memory load can be determined. In addition to the spatial search task, a commonly used spatial working memory task was administered, i.e., the Corsi Block-Tapping Task, which also requires participants to keep spatial information on-line in the visuospatial sketchpad. To study the effect of damage to the DLPFC, the PPC and the HF on these different processes, the spatial extent of the lesions was defined by transforming individual CT or MR images of the patients to a standard brain template, using lesion cost function masking (Brett, Leff, Rorden, \& Ashburner, 2001). Mapping the patients' brains with marked lesions to standard brain coordinates enables the comparison of lesions across patients and the correlation with the amount of damaged tissue in a brain area of interest with the observed behavior in standard neuropsychological tests.

We hypothesized that damage to the PPC and DLPFC impairs the ability to keep spatial information in working memory over a short time period, whereas damage to the HF might hamper the ability to keep spatial information in working memory over a more extended time period. These effects were hypothesized to be more profound for the right hemisphere than for the left hemisphere. Moreover, strategy use might be related to general "central-executive" aspects of working memory (Baddeley, 2002), possibly related to the dorsolateral prefrontal cortex.

\section{Methods}

\subsection{Participants}

Thirty stroke patients who were admitted to the University Medical Center Utrecht (UMCU) were examined. Sixteen patients had a lesion in the left hemisphere (LH), 13 patients had a lesion in the right hemisphere $(\mathrm{RH})$ and one patient had a large lesion in the RH accompanied by a small lesion in the $\mathrm{LH}$. All patients were examined at least 6 months after the stroke, were mobile and did not suffer from unilateral neglect or severe visual impairments at the time of testing (one patient reported mild hemianopsia, but after careful examination was found to see the display normally). Patients were all between 21 and 75 years of age and did not suffer from other neurological or psychiatric diseases. The study was approved by the medical ethics committee of the UMCU and written informed consent was obtained according to the declaration of Helsinki. We also examined 36 age- and education-matched, healthy control participants who were recruited through an advertisement in the local newspaper and were paid for their participation. Characteristics of the patients and comparison group are shown in Table 1. Handedness was assessed with a Dutch version of the Annett Handedness Inventory (Briggs \& Nebbs, 1975). Education level was measured using seven categories ( 1 being the lowest and 7 the highest; Hochstenbach, den Otter, \& Mulder, 2003). No differences were found between the three groups for education level $[F(2,62)=2.1]$, age $[F(2,63)=0.1]$, or gender distribution $\left[\chi^{2}(2)=4.4\right]$

Standard neuropsychological tests were used to assess overall intelligence and memory performance. Verbal intelligence was estimated with the Dutch version of the National Adult Reading Task (Schmand, Bakker, Saan, \& Louman, 1991); non-verbal intelligence with the 12-item short form of the Raven Advanced Progressive Matrices (Raven, Raven, \& Court, 1993). Verbal memory was assessed with the Dutch version of the Rey Auditory Verbal Learning Test (RAVLT, Rey, 1964). The Letter Number Sequencing task (WAIS-III) was used as an index of verbal working memory (Wechsler, 1987). No differences were found on any of the standard neuropsychological tests between the patients and controls, except on immediate reproduction of the RAVLT $[F(2,58)<7.1$, $p<0.01]$. Patients with a lesion in the RH or LH performed worse than the control participants $[t(48)=3.2, p<0.01, t(48)=2.8, p<0.01$, respectively]; whereas no difference was found between patients with a lesion in the $\mathrm{RH}$ or to the $\mathrm{LH}$ $[t(24)=0.2]$.

Table 1

Characteristics and neuropsychological test results of patients with lesions in the right $(\mathrm{RH})$ and in the left hemisphere ( $\mathrm{LH})$ and of control participants

\begin{tabular}{lccc}
\hline & RH $(n=14)$ & LH $(n=16)$ & Controls $(n=36)$ \\
\hline Age (years) & $57.8(3.1)$ & $57.8(2.8)$ & $56.9(1.8)$ \\
Education level (1-7) & $4.7(0.4)$ & $5.3(0.3)$ & $5.5(1.0)$ \\
Annett handedness & $15.2(4.2)$ & $17.8(2.9)$ & $15.3(2.2)$ \\
$\quad$ inventory (-24/24) & & & \\
Sex (m:f) & $12: 2$ & 11.5 & $20: 16$ \\
NLV-IQ & $107.5(6.1)$ & $105.6(5.6)$ & $107.2(3.7)$ \\
RAVLT: immediate recall & $36.5(2.8)^{*}$ & $37.5(3.1)^{*}$ & $46.9(1.7)$ \\
RAVLT: delayed recall & $8.5(1.1)$ & $7.8(1.5)$ & $9.6(0.6)$ \\
Raven APM (short form) & $7.0(0.9)$ & $8.0(0.7)$ & $12.0(2.8)$ \\
Letter number sequencing & $9.5(0.8)$ & $8.5(0.7)$ & $9.8(0.3)$ \\
$\quad$ task & & & \\
Corsi Block-Tapping Task & $45.8(4.6)$ & $43.6(3.5)$ & $42.4(2.5)$ \\
\hline
\end{tabular}

Note: NLV = Dutch version of the National Adult Reading Task, RAVLT = Rey Auditory Verbal Learning Test, Raven APM = Raven Advanced Progressive Matrices.

* Significant difference with the controls $(p<0.05)$. 


\subsection{Material and procedure}

\subsubsection{Corsi Block-Tapping task}

The Corsi Block-Tapping Task is a widely used neuropsychological test of spatial working memory (Berch, Krikorian, \& Huha, 1998; Kessels, Van Zandvoort, Postma, Kappelle, \& De Haan, 2000). A recently introduced sensitive measure of the Corsi task is used in the current experiment, which is the product of the total number of correct trials and the length of the largest sequence (Kessels et al., 2000).

\subsubsection{Box task}

In this spatial working memory task (Kessels et al., 2004; Van Asselen et al., 2005), pictures of closed boxes were randomly displayed on different locations within a square of $19 \times 19 \mathrm{~cm}$ on a $15 \mathrm{in}$. touch-sensitive LCD computer monitor. An easy-to-name, colored target object (e.g. ball, tomato) was presented (approximately $1 \mathrm{~cm} \times 1 \mathrm{~cm}$ ) at the bottom of the square. Participants were instructed to find the target object, which was hidden in one of the boxes and could not be seen when the boxes were closed. The boxes could be opened by touching them, after which either an empty box or the target object was shown at the same location. An empty box remained open for $2 \mathrm{~s}$, whereas the target object was shown until the participant initiated a new search. After successfully locating a target object, a new target appeared below the square. Importantly, the target object that was found before remained in the box where it was found until the end of the trial and no second object could be added to this box. Thus, the participant not only had to remember which box was searched, but also in which box a previous target object was found. After locating the second target object, a third target appeared below the square. This would continue until all boxes contained a target object. Which box was filled with an object was randomly determined and not dependent on the participants' response. The experiment began with two practice trials of three boxes, after which two trials of 4, 6 and 8 boxes were used. No time limit was set. A single search through the boxes in order to locate a target object took approximately $5-11 \mathrm{~s}$ and a trial $(4,6$ or 8 searches together) $20-88 \mathrm{~s}$, depending on the set size and number of errors. Fig. 1 shows an example of the layout of the Box task when making a search through 6 boxes.
Three dependent variables were included: (1) within-search errors were scored when a participant returned to an already opened box within a search; (2) between-search errors were scored when a participant returned to a box where a target object was found in one of the previous searches; (3) efficient use of strategy was defined by counting the number of times a participant started searching for a target object with a different box (within one trial). Strategy use is considered more efficient if a participant follows a predetermined search sequence, since this relieves memory load. Thus, a low score on the strategy index would indicate efficient use of strategy (see also Feigenbaum et al., 1996; Van Asselen et al., 2005).

\subsection{Lesion location}

CT or MR images were available for 26 patients (12 RH, $13 \mathrm{LH}$ and one patient with a lesion in both hemispheres). For five patients no digital scans were available, who were excluded from further lesion analyses. The Corsi Block-Tapping Task was not performed by one patient, and in another patient the Box task was not administered. In order to define the locations of the lesions, an experienced neurologist who was unaware of the clinical status of the patients, manually delineated for all patients the regions of interest (ROI) covering the lesion in all slices, using the MRIcro software (http://www.psychology.nottingham.ac.uk/staff/cr1/mricro.html). ROIs were saved as image volumes containing 1 for voxels inside the lesion area and 0 for voxels outside the lesion area. CT images were skull stripped by only including voxels with a CT-intensity value between 0 and 100. All images were then normalized to standard brain coordinates (MNI) according to the method described by Brett et al. (2001) using the software package Statistical Parametric Mapping (SPM2) (http://www.fil.ion.ucl.ac.uk/spm/software/spm2/). CT or MRI-scans were normalized to a T1-weighted template, using cost function masking with smoothed masks (FHWM $=8 \mathrm{~mm}$ ) based on the ROI (Fig. 2). By doing so, voxels in the lesioned tissue do not contribute to the calculation of the spatial transformation from the patients brain to MNI (standard brain) coordinates, which results in a standardized map of the pathological brain images (Brett et al., 2001). Finally, the ROI images and CT/MRI scans were resliced with a $1 \mathrm{~mm} \times 1 \mathrm{~mm} \times 1 \mathrm{~mm}$ voxel size. The lesion size of each patient was calculated

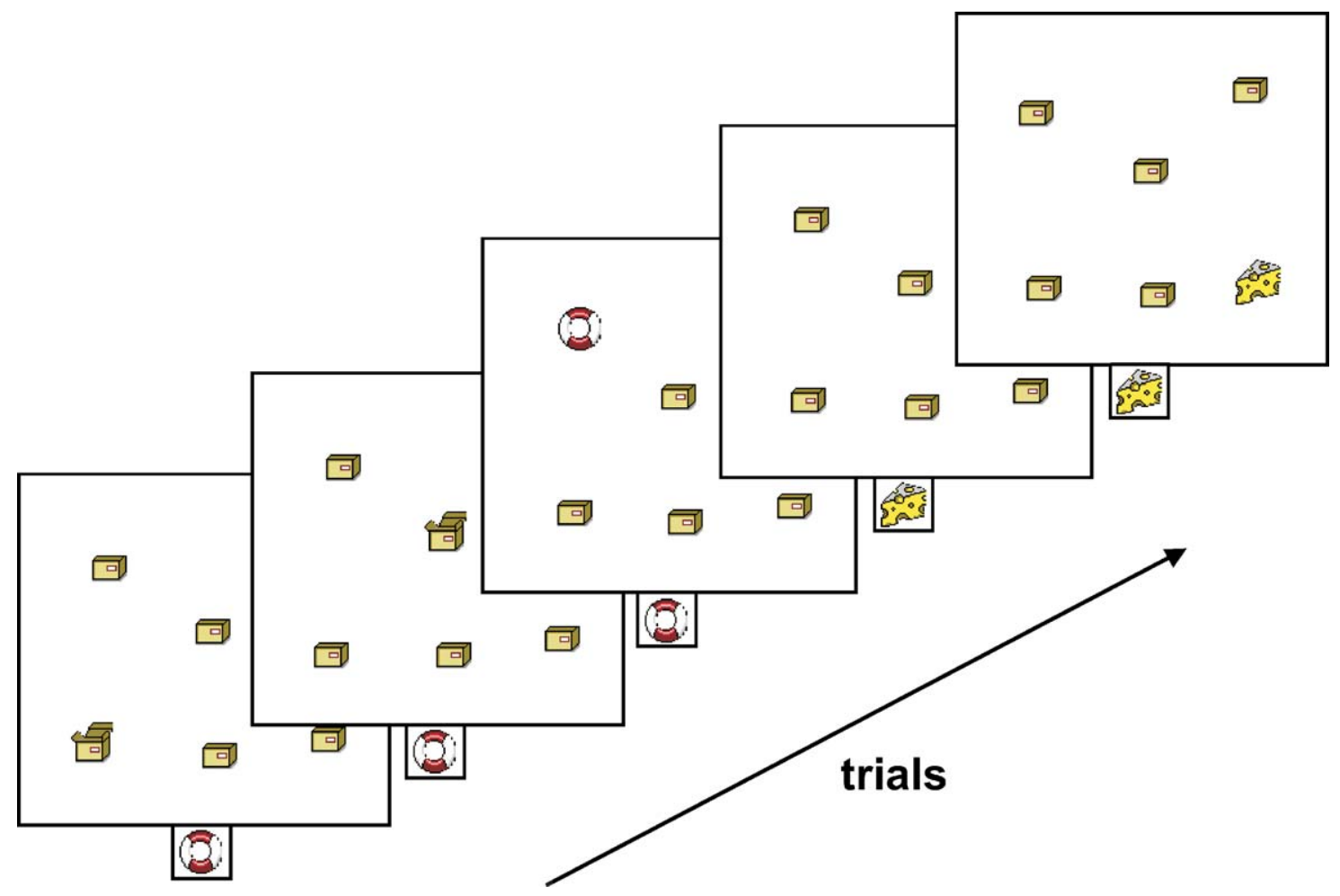

Fig. 1. Example of the display of a search through 6 boxes, including presentation of two different target objects. 

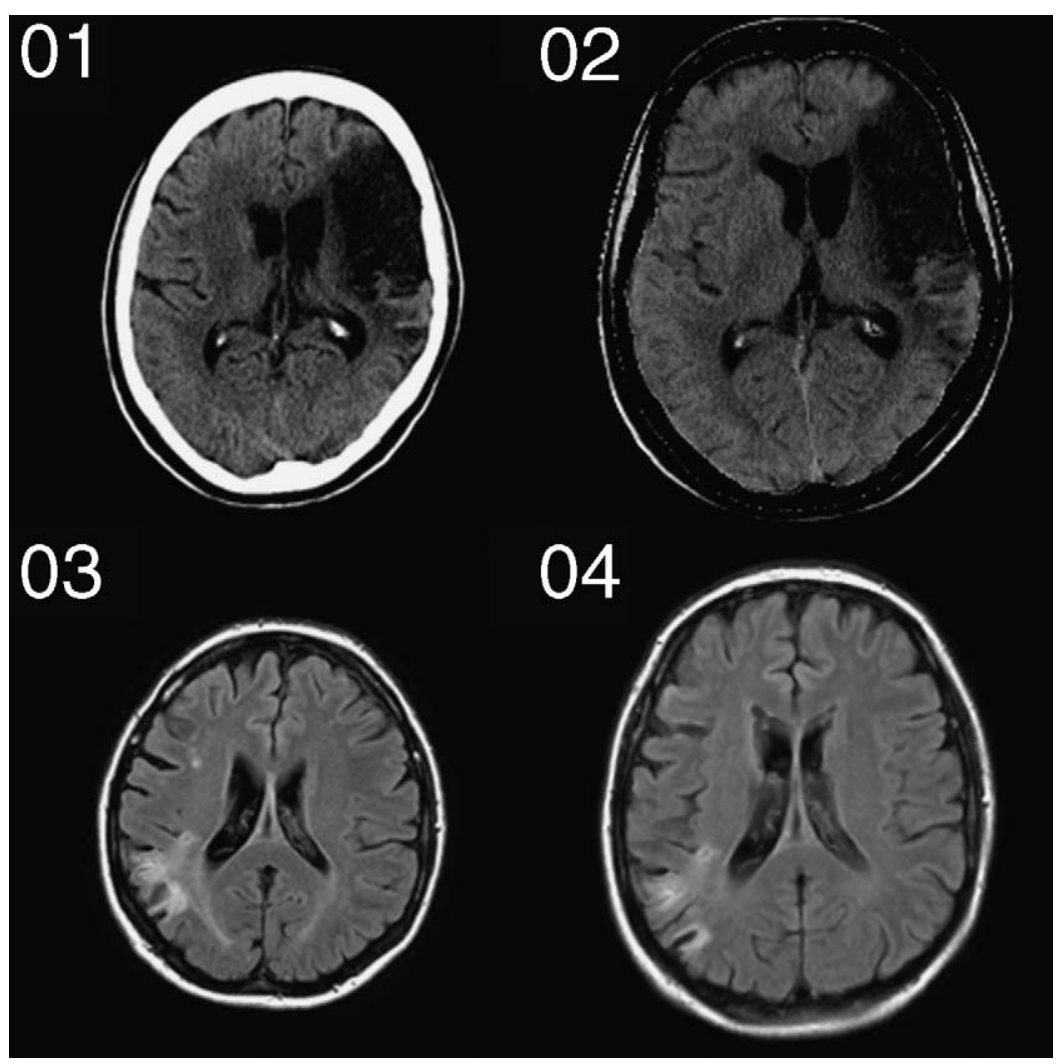

Fig. 2. An example of a CT scan before (01) and after (02) transforming it to standard brain template and an example of an MRI scan before (03) and after (04) transforming it to standard brain template.

as defined by the number of voxels that were above threshold (threshold $=0.5$ ), since due to interpolation artefacts at the reslicing stage voxels in the normalized images could have values between 0 and 1 . Importantly, no difference was found between the lesion sizes of the group of patients with a lesion in the RH and those with a lesion in the LH [t(24) $=1.3]$. Normalized brain scans including the lesions are shown in Fig. 3 for each patient separately.

Volumes of interest (DLPFC, PPC and HF) were used that were established by probability density estimates of locations from the BrainMap database (Nielsen \& Hansen, 2002). For each patient it was determined whether the lesion included one or more of these areas and subsequently the size of the lesion in the target area was calculated as defined by the number of voxels of the lesion that were above threshold and located within the target region divided by the total number of voxels in the target region (lesion overlap ratio). Twelve patients had a lesion in the DLPFC (Fig. 3: nos. 02, 06, 07, 08, 09, 12, 13, 14, 18, 20, 24, 26), 16 patients had a lesion in the HF (Fig. 3: nos. 01, 04, 05, 06, 07, 10, 11, $12,13,15,17,18,22,23,24,25)$ and five patients had a lesion in the right PPC (Fig. 3: nos. 05, 07, 10, 11, 12). Since there was only one patient with damage to the left PPC, the effect of damage to this brain area on spatial working memory was not studied.

\subsection{Analyses}

First, to examine general lateralization effects, performances of LH and RH patients were compared with the performances of control participants (overall hemispheric differences) by means of a one-way ANOVA (Corsi task) (RH, LH, controls) or a $3 \times 3$ Repeated Measures analysis (within- and between-search errors) with between-subject variable group (RH, LH, controls) and withinsubject variable set size (4, 6, 8 boxes). For the strategy-index of the Box task lateralization effects were analysed by means of a $2 \times 3$ Repeated Measures analysis with within-subject variable set size ( 6 and 8 boxes) and betweensubject variable group (RH, LH, controls).

Second, to study the effect of damage to a specific target area (HF, DLPFC or PPC) in either the LH or RH on the within- and between-search errors (analyses of specific brain areas), $3 \times 3$ Repeated Measures analyses were performed with between-subject variable group (RH, LH, controls) and within-subject variable set size ( 4,6 and 8 boxes). ANOVA was performed to study the effect of damage to a specific target area (RH, LH, controls) on the Corsi Block-Tapping task. Patients with limited damage to the target region were included in group 1 or 2 .

Third, to study the influence of the extent of damage within a particular area on the different spatial memory tasks, one-tailed Pearson or Spearman correlations were performed between the lesion overlap ratio of a target area and performance on the working memory tasks (correlations).

\section{Results}

\subsection{Corsi Block-Tapping task}

\subsubsection{Overall hemispheric differences}

No difference in the total score between $\mathrm{RH}$ patients, $\mathrm{LH}$ patients and the control group (Fig. 4) was found $[F(2,65)=$ $0.26]$.

\subsubsection{Analyses of specific brain areas}

ANOVA did not show group differences for patients with damage to the DLPFC $[F(2,46)=0.1]$, the PPC $[t(39)=0.3]$, or the $\operatorname{HF}[F(2,49)=1.0]($ Fig. 5).

\subsubsection{Correlations}

Significant negative correlations were found between performance on the Corsi Block-tapping task and the lesion overlap ratio of the right DLPFC $[r=-0.43, p<0.05]$ and the right PPC $[r=-0.42, p<0.05]$. No correlations were found 


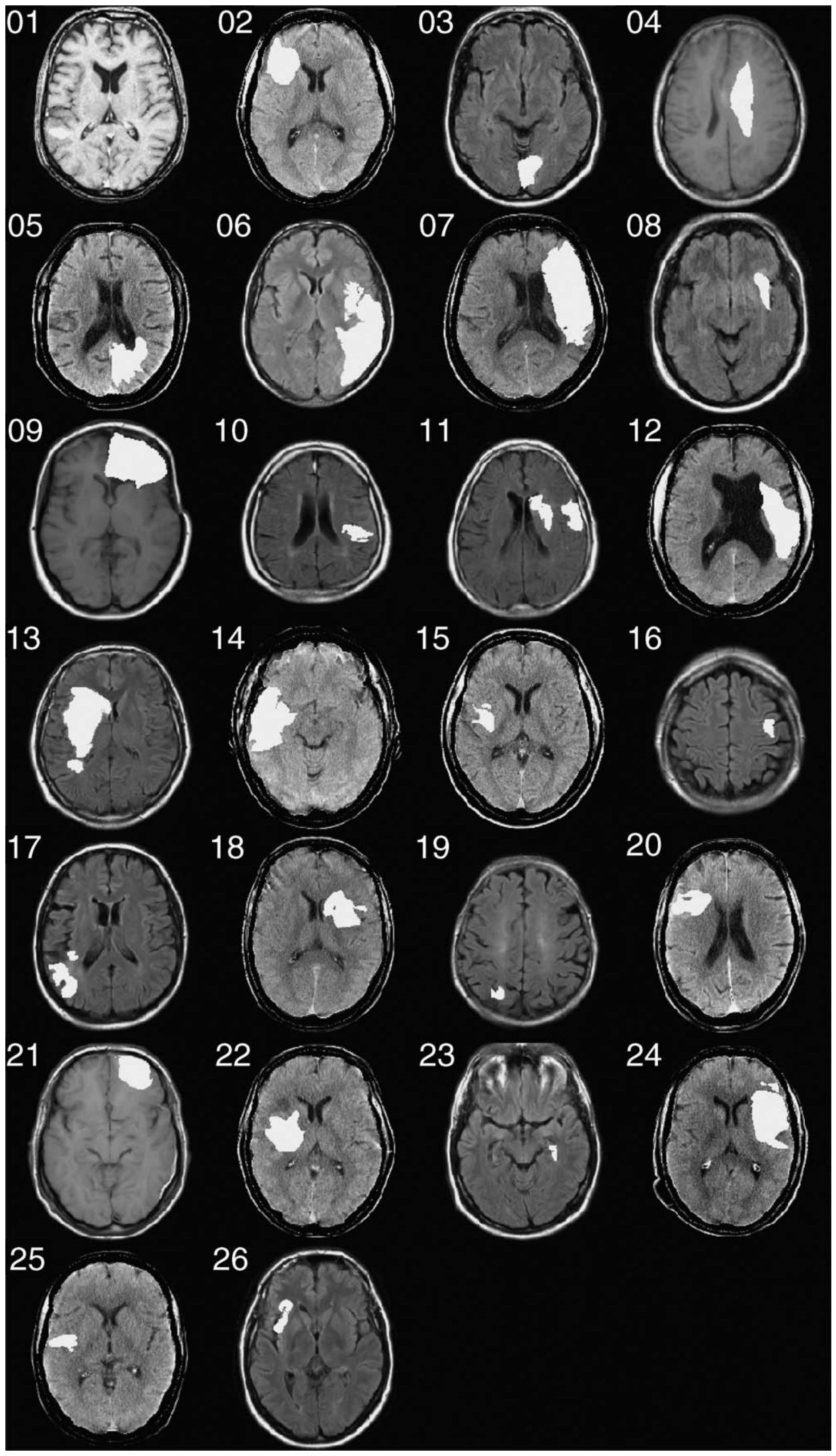

Fig. 3. CT and MRI scans of the 26 patients after transformation to standard brain template. 


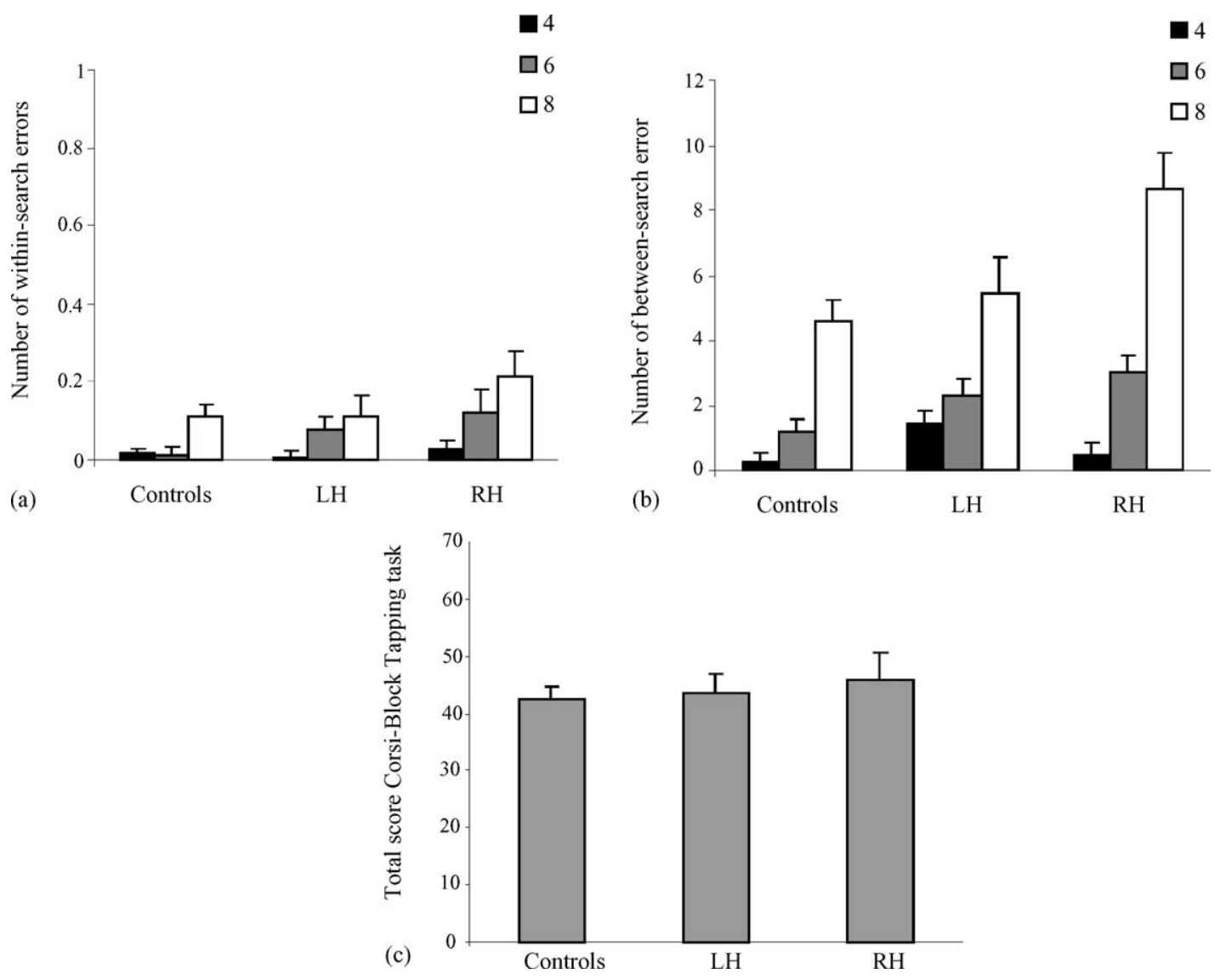

Fig. 4. (a) The average number of within-search errors (set sizes $4,6,8$ ) of the patients with a lesion in the right (RH) or left hemisphere (LH) and control participants; (b) average number of between-search errors (set sizes $4,6,8$ ) of the patients with a lesion in the right or left hemisphere and control participant; (c) total score on the Corsi Block-Tapping task of the patients with a lesion in the right hemisphere or left hemisphere and control participants.

between performance on the Corsi Block-Tapping task and the left DLPFC $(r=0.05)$, or the HF $(r<0.25)$. It should be noted, that of the six patients with damage to the right DLPFC, two patients had severe damage (Fig. 3: nos. 07+24), and four patients had only minor damage. Importantly, the patients with large lesions were severely impaired, whereas the others were not. Since the patients with minor damage to the right DLPFC were unimpaired on the Corsi test, no difference was found in a group comparison. Similarly, of the five patients with damage to the right PPC, only one patient had a large lesion (Fig. 3: no. $07)$ and this patient was clearly impaired.

\subsection{Box task: within-search errors}

\subsubsection{Overall hemispheric differences}

More within-search errors (Fig. 4) were made with increasing set size $[F(2,126)=13.6, p<0.001]$. A test of withinsubject contrasts showed that more errors were made with increasing set size $(4-6$ boxes $[F(1,63)=7.3, p<0.01]$ and $8-6$ boxes $[F(1,63)=5.7, p<0.05])$. Moreover, a main effect for group was found $[F(2,63)=3.7, p<0.05]$. Contrast analysis showed that RH patients made significantly more errors than the control participants $[p<0.01]$, whereas no difference was found between LH patients and the control group.
No interaction effect between group and set size was found $[F(4,126) \leq 1.1]$.

\subsubsection{Analyses of specific brain areas}

Repeated measures analyses including patients with damage to the DLPFC revealed a significant effect for set size $[F(2,90)=4.4, p<0.015]$, indicating that more within-search errors were made in the condition with 6 boxes than the condition with 4 boxes $[F(1,46)=11.3, p<0.005]$, whereas no difference was found between the condition with 8 boxes and the condition with 6 boxes $[F(1,45)=0.8]$ (Fig. 5). No significant effect was found for the variable group $[F(2,45)=2.6]$, nor was an interaction effect between group and set size found $[F(4,90)=2.6]$.

Repeated Measures analyses including the patients with damage to the right HF, patients with damage to the left $\mathrm{HF}$ and controls demonstrated a significant effect for set size $[F(2,96)=14.2, p<0.001]$, indicating that more errors were made with increasing set size $(4-6$ boxes $[F(1,50)=4.4$, $p<0.05]$, and $6-8$ boxes $[F(1,48)=11.6, p<0.001])$. However, no effect for group $[F(2,48)=1.2]$ was found, nor an interaction effect between group $\times$ set size $[F(4,96)=0.8]$.

Similarly, Repeated Measures analyses including patients with damage to the right PPC and controls revealed a significant effect for set size $[F(2,39)=11.5, p<0.001]$, but not for group 

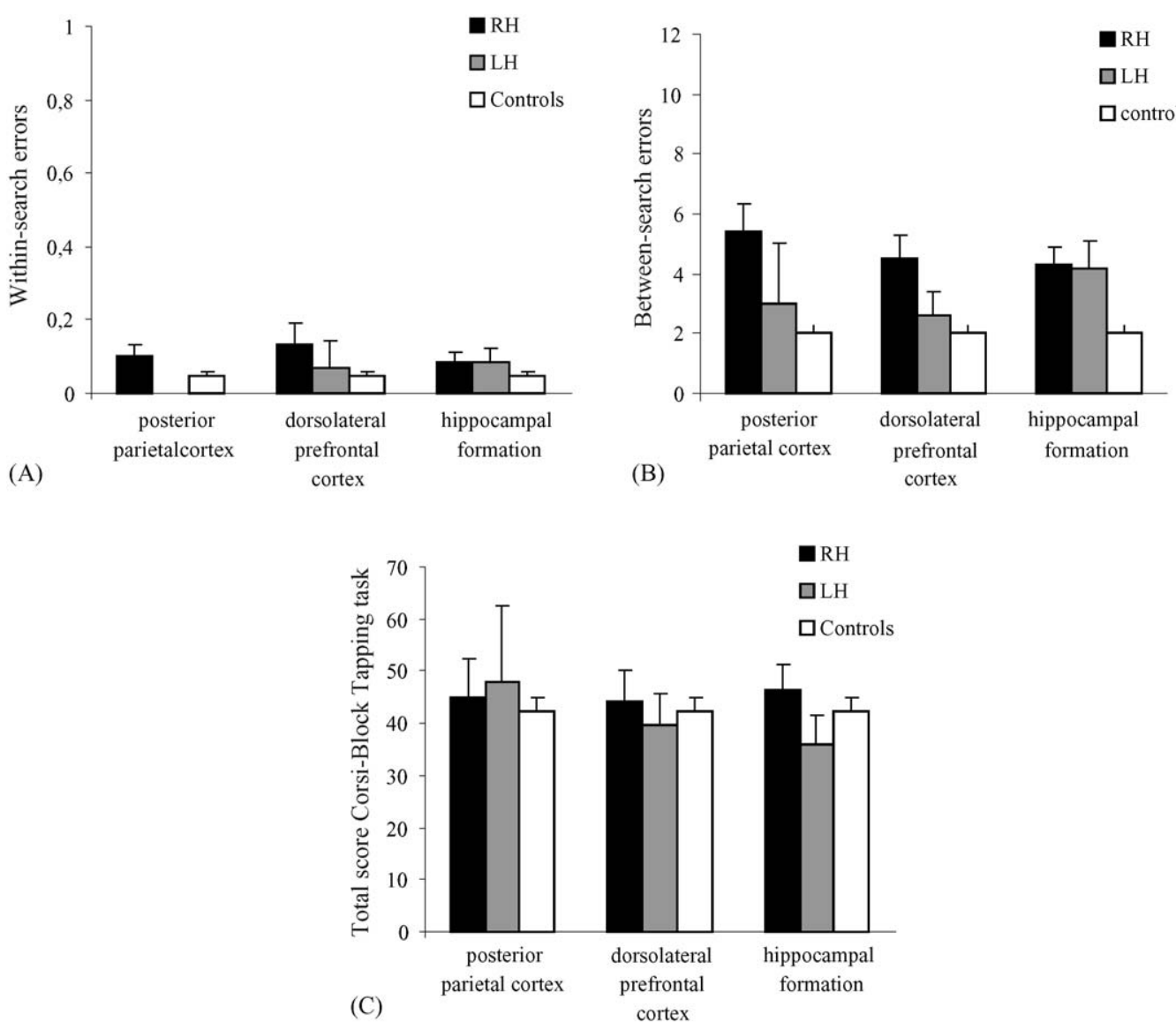

Fig. 5. (a) Average number of within-search errors (set sizes 4,6,8) of the patients with a lesion in the dorsolateral prefrontal cortex, posterior parietal cortex and hippocampal formation (left and right hemisphere lesions separately) and control participants; (b) average number of between-search errors (set sizes 4, 6, 8) of the patients with a lesion in the dorsolateral prefrontal cortex, posterior parietal cortex and hippocampal formation (left and right hemisphere lesions separately) and control participants; (c) total score on the Corsi Block-Tapping task of the patients with a lesion in the dorsolateral prefrontal cortex, posterior parietal cortex and hippocampal formation (left and right hemisphere lesions separately) and control participants.

$[F(1,39)=2.5]$. No interaction effect between group $\times$ set size $[F(2,78)=1.7]$ was found.

\subsubsection{Correlations}

No significant correlations were found between the lesion overlap ratio of the left and right DLPFC $(r<0.29)$, the right PPC $(r<0.10)$, or the left and right HF $(r<0.17)$ and the mean number of within-search errors of the 4, 6 and 8 boxes.

\subsection{Box task: between-search errors}

\subsubsection{Overall hemispheric differences}

For the between-search errors (Fig. 4) a significant main effect was found for set size $[F(2,126)=74.2, p<0.001]$. A test of within-subject contrasts showed that the errors increased as a result of increasing set size $(4-6$ boxes $[F(1,63)=44.2$, $p<0.001]$ and $6-8$ boxes $[F(1,63)=64.4, p<0.001])$. Additionally, a significant effect for group was found $[F(2,63)=5.1$, $p<0.01]$. Contrast analysis showed that $\mathrm{RH}$ patients made more between-search errors than control participants $(p<0.01)$, whereas no difference was found between the LH patients and control participants. Moreover, an interaction effect for group $\times$ set size $[F(4,126)=3.6, p<0.01]$ was found. Tests of within-subjects contrasts showed a significant group $\times$ set size effect for the 4 and 6 boxes $[F(2,63)=5.6, p<0.01]$, but not the 6 and 8 boxes $[F(2,63)=2.1]$. The $2 \times 3$ repeated measures analysis showed that RH patients were more impaired by the increasing set size than the comparison group $[F(2,96)=7.6$, $p<0.01]$. The same analysis comparing LH patients with controls did not reveal a significant interaction effect $[F(2,100)=$ $0.04]$

\subsubsection{Analyses of specific brain areas}

Fig. 5 shows the between-search errors of the patient groups with damage to the PPC, the DLPFC and the HF (left and right separately). A significant effect was found for group, revealing a difference between right DLPFC patients, left DLPFC patients and controls $[F(2,45)=3.9, p<0.05]$. Contrast analysis indicated that patients with a lesion in the right DLPFC performed worse than the controls $(p<0.01)$, whereas no difference was found between patients with a lesion in the left DLPFC and controls. A significant effect for set size was found $[F(2,90)=25.8, p<0.001]$, indicating that the errors increased as a result of increasing set size $(4-6$ boxes $[F(1,46)=26.3$, $p<0.001]$ and $6-8$ boxes $[F(1,46)=18.9, p<0.001])$. Additionally, a significant interaction effect between group $\times$ set size was 
Table 2

The mean total scores of the patients with a lesion in the right $(\mathrm{RH})$ and left hemisphere $(\mathrm{LH})$ and the controls of the 6 and 8 box conditions separately on the strategy index

\begin{tabular}{llllll}
\hline & \multicolumn{2}{l}{6 boxes } & & \multicolumn{2}{l}{8 boxes } \\
\cline { 2 - 3 } \cline { 5 - 6 } & Mean & S.E. & & Mean & S.E. \\
\hline RH $(n=14)$ & 3.9 & 0.2 & & 4.6 & 0.2 \\
LH $(n=16)$ & 3.7 & 0.2 & & 4.7 & 0.2 \\
Controls $(n=36)$ & 3.4 & 0.1 & & 4.7 & 0.2 \\
\hline
\end{tabular}

found $[F(4,90)=3.5, p<0.05]$. Separate $2 \times 3$ Repeated Measures analyses indicated that right DLPFC patients deteriorated more than the controls with increasing set size $[F(2,80)=3.3$, $p<0.05]$, as well as the left DLPFC patients $[F(2,80)=3.1$, $p<0.05]$.

Patients with a lesion in the right PPC performed worse than control participants $[F(1,39)=15.6, p<0.001]$. A significant effect for set size was found $[F(2,78)=69.0, p<0.001]$, indicating that the errors increased as a result of increasing set size (4-6 boxes $[F(1,59)=19.1, p<0.001]$ and $6-8$ boxes $[F(1,57)=15.7$, $p<0.001])$. Additionally, a group $\times$ set size effect was found, revealing that right $\mathrm{PPC}$ patient deteriorated more with increasing set size than the healthy controls $[F(2,78)=12.6$, $p<0.001]$.

A group effect was found between patients with a lesion in the right $\mathrm{HF}$, patients with a lesion in the left $\mathrm{HF}$, and controls $[F(2,48)=7.4, p<0.005]$. Contrast analysis showed that patients with a lesion in the right HF performed worse than controls $(p<0.005)$, as well as patients with a lesion in the left HF $(p<0.05)$. Additionally, a significant effect for set size was found $[F(2,96)=94.2, p<0.001]$, indicating that the errors increased as a result of increasing set size $(4-6$ boxes $[F(1,50)=37.0$, $p<0.001]$ and $6-8$ boxes $[F(1,48)=83.4, p<0.001])$. Finally, a group $\times$ set size effect was found $[F(4,96)=6.8, p<0.001]$. Performance of $\mathrm{RH}$ patients deteriorated more with increasing set size $[F(2,88)=8.1, p<0.005]$, as well as performance of $\mathrm{LH}$ patients $[F(2,78)=8.2, p<0.005]$.

\subsubsection{Correlations}

A significant correlation was found between the mean number of between-search errors of the three conditions (set size 4, 6, 8 ) and the lesion overlap ratio of the right DLPFC $[r=0.56$, $p<0.005]$ and the right PPC $[r=0.42, p<0.05]$. No correlations were found between the right and left $\mathrm{HF}(r<0.16)$ and the mean number of between-search errors over the conditions with 4, 6 and 8 boxes. A negative correlation was found with the left DLPFC $[r=-0.36, p<0.05]$.

\subsection{Strategy use}

\subsubsection{Overall hemispheric differences}

No difference in strategy-index (Table 2) was found between $\mathrm{RH}$ patients or $\mathrm{LH}$ patients and controls $[F(1,62)=0.7]$. However, a significant effect was found for set size $[F(1,62)=43.8$, $p<0.001]$, indicating that strategy index increased with more boxes.

\subsubsection{Analyses of specific brain areas}

ANOVA revealed no significant difference with respect to the involvement of the DLPFC in the use of a strategy between patients with damage to the right DLPFC, patients with damage to the left DLPFC, and control participants $[F(2,44)=2.0]$.

\subsubsection{Correlations}

No correlation was found between the extent of damage to either the left or the right DLPFC and the strategy index $(r<0.31)$. Moreover, to analyse whether applying a strategy indeed resulted in fewer between-search errors the correlation between the strategy index and the within- and between-search errors for the 6 and 8 box conditions was calculated based on the group as a whole (patients and control participants). A significant correlation was found for the between-search errors $(r=0.28$, $p \leq 0.01)$, but not the within-search errors $(r=0.15)$. However, when performance of the patients and controls was analysed separately no correlation was found with both within- $(r<0.16)$ and between-search errors $(r<0.25)$.

\subsection{Lesion size and performance}

In order to study the effect of lesion size on performance on the different tasks, correlations were calculated between the lesion size in the right or left hemisphere and the different dependent variables. Only a single significant correlation was found between lesion size in the right hemisphere and the number of between-search errors in the condition with 6 boxes $(r=0.44$, $p<0.05)$. All of the other correlations were nonsignificant. This could be taken as an indication that in general a larger lesion does not necessarily implies lower performance.

\section{Discussion}

The aim of the current study was to examine in more detail the neural correlates of spatial working memory in a group of stroke patients. The Corsi Block-Tapping task is a commonly used test of spatial working memory, which requires participants to passively keep spatial information in working memory over a very short period of time without any kind of manipulation. The current study revealed no difference in performance on this task between patients with a lesion in either the LH or the RH and control participants. However, when performance was correlated with damage to the right DLPFC and the right PPC, a significant correlation was found, indicating that these areas serve to keep a sequence of locations in working memory. Damage to the left DLPFC or the HF did not impair this aspect of spatial working memory.

The spatial search task enabled us to examine various aspects of spatial working memory, that is, within-search errors, between-search errors and use of strategy. Patients with damage to the RH made more within-search errors than controls, whereas no difference was found between patients with a lesion in the LH and controls. Similar to the Corsi Block-Tapping Task, within-search errors reflect the ability to keep a sequence of locations in spatial working memory over a very short time period. Although previous studies have shown mixed results concerning 
lateralization effects for within-search errors (Miotto et al., 1996; Owen et al., 1990), the current study provides evidence that the RH is more specialised in this aspect of working memory. Importantly, this is not a result of a difference in the size of the lesions in the $\mathrm{LH}$ and $\mathrm{RH}$ or a difference in general cognitive ability and memory function as was assessed with standard neuropsychological tests. Looking at the relation between the number of within-search errors and damage to specific brain areas, we found no increase in within-search errors due to damage to the right or left DLPFC, the right PPC or the right or left HF. Thus, in contrast to the Corsi Block-Tapping task, damage to the right PPC and right DLPFC did not lead to more within-search errors. It should be noted, however, that only few within-search errors were made by both the patients and the healthy controls, indicating that this aspect of the search task might not be very sensitive.

With respect to the between-search errors we found that patients with damage to the RH, but not those with lesions in the LH, were impaired in comparison to the controls. This lateralization effect supports previous findings of $\mathrm{RH}$-specialisation for spatial aspects of working memory (Feigenbaum et al., 1996; Miotto et al., 1996; Nelson et al., 2000). Moreover, as expected, overall performance deteriorates with increasing set size. This deterioration, however, was stronger in the case of RH lesions than of LH lesions. Furthermore, it was found that patients with damage specifically to the right DLPFC, the right PPC, or the left or right HF made more between-search errors than control participants. Moreover, a significant correlation of the betweensearch errors with lesion size in both the right DLPFC and the right PPC was found, but not with lesion size in the HF. This can be explained by the fact that the HF is less crucial for spatial working memory than the right PPC and the right DLPFC.

When interpreting these results it is important to consider that in order to avoid between-search errors, spatial information has to be kept in memory over a longer time period (possibly reflecting transfer into long-term memory), whereas the Corsi Block-Tapping Task requires spatial information to be kept in memory over a short time period. Interestingly, this would suggest that the DLPFC and the PPC are essential for keeping spatial information in memory over a short time period, whereas the HF is involved in the transfer from working memory into long-term memory. However, it cannot be excluded that the HF is also involved in the reverse process, i.e. transferring spatial information from long-term memory into working memory, in order to use this information during a search. The current results are in line with observations from neuroimaging and animal studies suggesting that brain areas that are active in spatial working memory, including the DLPFC, PPC and HF, serve different roles (Constantinidis \& Wang, 2004; Friedman \& GoldmanRakic, 1994; Glabus et al., 2003; Inoue, Mikami, Ando, \& Hideo, 2004). However, the current study does not allow a conclusive decision about the nature of spatial information that is processed, i.e. egocentric (viewer-dependent) or allocentric information (viewer-independent). That is, the Box task can be solved with solely egocentric information (remembering the position of the boxes in relation to the viewer), but allocentric information can be used as well (remembering the positions of the boxes in relation to external landmarks in the test room). Future research will have to focus on using tasks that are more strict measures of either egocentric or allocentric spatial working memory.

Finally, an index was calculated assessing the use of an efficient strategy to remember the target locations and the previously opened empty boxes. Using a strategy could therefore help to overcome memory problems, and thus would be especially important with larger set sizes. No difference in the use of a strategy was found between the entire patient group and the control participants. However, the strategy-index may not be a very sensitive measure, since a correlation between the strategy index and the within- and between-search errors was only found when both the patients and controls were taken together.

It should be noted that the results of this study are not caused by a general right hemisphere effect, in which larger lesions in the right hemisphere lead to larger impairments, irrespective of the lesion location. That is, no correlations, except one (larger lesions in the right hemisphere resulted in more betweensearch errors in the condition with 6 boxes) were found between the size of the lesions and performance on the dependent variables (between-search errors, within-search errors and Corsi Block-Tapping task). This indicates that a larger lesion in the right hemisphere does not necessarily imply lower performance. Additionally, although a general right hemisphere lateralisation effect was found, clear selective effects were found within the right hemisphere, e.g. damage to the right DLPFC and right PPC but not the right hippocampal formation results in more within-search errors.

The Corsi Block-Tapping Task revealed no lateralization effects, whereas impairments were found when specific brain areas were studied. This underlines the disadvantage of studying heterogenic groups of patients. By defining the exact locations of the lesions of our patients, we were able to study the effect of damage to specific areas in the brain on spatial working memory. Moreover, defining the sizes of the lesions in specific areas of the brain enabled us to calculate correlations between the extent of damage and performance on the working memory tasks. This is an even more accurate measure, since impairments are expected to be larger when more tissue is damaged. Additionally, this partly resolves the problem of overlap of lesion sites in patients. That is, patients can have a lesion that involves more than one target area. In the current study this problem was limited, since only four patients had damage to two target areas, and one patient had damage to three target areas. Usually damage to one of the target areas was significantly larger than damage to the other target area, which is noted in the correlations. A possible limitation of the current study, however, is that MRI and CT scans were not made at the moment of testing. Therefore, the size of the lesions may have changed in the period between making the scan and testing the patient or new lesions could have developed. Although the current study clearly underlines the importance of exploring new methods of studying the effect of brain damage on cognitive processes such as spatial memory, it should be noted that while the present results show clear relations between brain areas and spatial working memory function, they should 
be studied in larger samples. In order to allow a more explorative study of the neural correlates of spatial working memory, further research should focus on testing larger patient groups.

In conclusion, the current study demonstrated that in particular the right DLPFC and the right PPC are involved in keeping spatial information in memory over a short time period, as was assessed with the Corsi Block-Tapping task. Moreover, both right and left $\mathrm{HF}$ seem to have a fundamental role in maintaining spatial information in working memory over an extended time period, as was assessed with the between-search errors.

\section{References}

Abrahams, S., Morris, R. G., Polkey, C. E., Jarosz, J. M., Cox, T. C. S., Graves, M., et al. (1999). Hippocampal involvement in spatial and working memory: A structural MRI analysis of patients with unilateral mesial temporal lobe sclerosis. Brain and Cognition, 41, 39-65.

Abrahams, S., Pickering, A., Polkey, C. E., \& Morris, R. G. (1997). Spatial memory deficits in patients with unilateral damage to the right hippocampal formation. Neuropsychologia, 35, 11-24.

Baddeley, A. D. (2002). Is working memory still working? European Psychologist, 7, 85-97.

Berch, D. B., Krikorian, R., \& Huha, E. H. (1998). The Corsi Block-Tapping Task: Methodological and theoretical considerations. Brain and Cognition, 38, 317-338.

Bohbot, V. D., Jech, R., Růžička, E., Nadel, L., Kalina, M., Stěpánková, K., et al. (2002). Rat spatial memory tasks adapted for humans: Characterization in subjects with intact brain and subjects with medial temporal lobe lesions. Physiological Research, 51, 49-64.

Brett, M., Leff, A. P., Rorden, C., \& Ashburner, J. (2001). Spatial normalization of brain images with focal lesions using cost function masking. Neuroimage, 14, 486-500.

Briggs, G. G., \& Nebbs, R. D. (1975). Patterns of hand preference in a student population. Cortex, 11, 230-238.

Constantinidis, C., \& Wang, X. J. (2004). A neural circuit basis for spatial working memory. Neuroscientist, 10, 553-656.

Feigenbaum, J. D., Polkey, C. E., \& Morris, R. G. (1996). Deficits in spatial working memory after unilateral temporal lobectomy in man. Neuropsychologia, 34, 163-176.

Fletcher, P. C., \& Henson, R. N. A. (2001). Frontal lobes and human memory: Insights from functional neuroimaging. Brain, 124, 849-881.

Friedman, H. R., \& Goldman-Rakic, P. S. (1994). Coactivation of prefrontal cortex and inferior parietal cortex in working memory tasks revealed by 2DG functional mapping in the rhesus monkey. Journal of Neuroscience, $14,2775-2788$.

Glabus, M. F., Horwitz, B., Holt, J. L., Kohn, P. D., Gerton, B. K., Gallicott, J. H., et al. (2003). Interindividual differences in functional interactions among prefrontal, parietal and parahippocampal regions during working memory. Cerebral Cortex, 13, 1352-1361.

Goldman-Rakic, P. S. (1988). Topography of cognition: Parallel distributed networks in primate association cortex. Annual Review of Neuroscience, $11,137-156$.
Hochstenbach, J. B., den Otter, R., \& Mulder, T. W. (2003). Cognitive recovery after stroke: A 2-year follow-up. Archives of Physical Medicine and Rehabilitation, 84, 1499-1504.

Inoue, M., Mikami, A., Ando, I., \& Hideo, T. (2004). Functional brain mapping of the macaque related to spatial working memory as revealed by PET. Cerebral Cortex, 14, 106-119.

Jonides, J., Smith, E. E., Koeppe, R. A., Awh, E., Minohsima, S., \& Mintun, M. A. (1993). Spatial working memory in humans as revealed by PET. Nature, 363, 623-625.

Kessels, R. P. C., Hendriks, M. P. H., Schouten, J., Van Asselen, M., \& Postma, A. (2004). Spatial memory deficits in patients after unilateral selective amygdalohippocampectomy. Journal of International Neuropsychology, 10, 1-6.

Kessels, R. P. C., Van Zandvoort, M. J. E., Postma, A., Kappelle, L. J., \& De Haan, E. H. F. (2000). The Corsi Block-Tapping Task: Standardization and normative data. Applied Neuropsychology, 7, 252-258.

Miotto, E. C., Bullock, P., Polkey, C. E., \& Morris, R. G. (1996). Spatial working memory and strategy formation in patients with frontal lobe excisions. Cortex, 32, 613-630.

Nelson, C. A., Monk, C. S., Lin, J., Carver, L. J., Thomas, K. M., \& Truwit, C. L. (2000). Functional neuroanatomy of spatial working memory in children. Developmental Psychology, 36, 109-116.

Nielsen, F. A., \& Hansen, N. K. (2002). Automatic anatomical labeling of Talairach coordinates and generation of volumes of interest via the BrainMap database. NeuroImage, 16(Suppl. 2).

Owen, A. M., Downes, J. J., Saahakian, B. J., Polkey, C. E., \& Robbins, T. W. (1990). Planning and spatial working memory following frontal lobe lesions in man. Neuropsychologia, 28, 1021-1034.

Owen, A. M., Stern, C. E., Look, R. B., Tracey, I., Rosen, B. R., \& Petrides, M. (1998). Functional organization of spatial and nonspatial working memory processing within the human lateral frontal cortex. Proceedings of the National Academic of Science of the United States of America, 95, $7721-7726$.

Raven, J. C., Raven, J., \& Court, J. H. (1993). Manual for Raven's progressive matrices and vocabulary scales. Oxford: Oxford Psychologists Press.

Rey, A. (1964). L'examen clinique en psychologie [Clinical assessment in psychology]. Paris: Presses Universitaires de France.

Schmand, B., Bakker, D., Saan, R., \& Louman, J. (1991). De Nederlandse Leestest voor Volwassenen: Een maat voor het premorbide intelligentieniveau [The Dutch adult reading test: A measure of premorbid intelligence]. Tijdschrift voor Gerontologie en Geriatrie, 22, 15-19.

Smith, E. E., \& Jonides, J. (1997). Working memory: A view from neuroimaging. Cognitive Psychology, 33, 5-42.

Van Asselen, M., Kessels, R. P. C., Wester, A., \& Postma, A. (2005). Spatial working memory and contextual cueing in patients with Korskoff amnesia. Journal of Clinical and Experimental Neuropsychology, 27, 5.

Walter, H., Bretschneider, V., Grön, G., Zurowski, B., Wunderlich, A. P., Tomczak, R., et al. (2003). Evidence for quantitative domain dominance for verbal and spatial working memory in frontal and parietal cortex. Cortex, 39, 897-911.

Wechsler, D. (1987). Wechsler memory scale - Revised manual. San Antonio, TX: Psychological Corporation.

Wilson, F. A. W., Scalaidhe, S. P. O., \& Goldman-Rakic, P. S. (1993). Dissociation of object and spatial processing domains in primate prefrontal cortex. Science, 260, 1955-1958. 